\title{
Awareness And Practices Towards Nutraceuticals Among Medical Practitioners of A Tertiary Care Teaching Hospital in South India - A Pilot Study
}

\author{
Navyashree $\mathrm{R}^{1}$, Mohandas Rai $^{2 *}$, Arun Ravindran $^{3}$ \\ ${ }^{\text {I}}$ (Postgraduate, Department Of Pharmacology, A.J Institute Of Medical Sciences And Research Centre. \\ Mangaluru, Karnataka, India) \\ $2^{2 *}$ (Professor And Head, Department Of Pharmacology, A.J Institute Of Medical Sciences And Research Centre. \\ Mangaluru, Karnataka, India) \\ ${ }^{3}$ (Assistant Professor, Department Of Pharmacology, A.J Institute Of Medical Sciences And Research Centre. \\ Mangaluru, Karnataka, India
}

\begin{abstract}
Nutraceuticals' refer to food or parts of foods that provide medical or health benefits including prevention and treatment of disease. Nutraceuticals are increasingly being prescribed in day to day practice. There is a dearth of studies outlining the use and prescription of nutraceuticals in the Indian population. This pilot study aims at assessing the knowledge, attitude and practices among registered medical practitioners at a tertiary care teaching hospital in South India.

A self structured, questionnaire based pilot study was conducted among medical practitioners. After obtaining informed consent, participants were asked to complete the questionnaire and information provided was analysed using Graphpad Prism Version 6.0. All data were expressed as percentages.

50 questionnaires were completed and returned by the approached registered medical practitioners. $76 \%$ of the study participants were aware of the term nutraceuticals. The percentage of respondants who said that nutraceuticals should be sold only on prescription of registered medical practitioners are 78\%. Among all the respondents $54 \%$ of them prescribe nutraceuticals in their daily practice.

Nutraceuticals are increasingly being prescribed by medical practitioners but there is need for further study to analyse the attitude and practices towards them.
\end{abstract}

Keywords: Medical Practitioners, Nutraceuticals, Questionnaire

\section{Introduction}

The words quoted by the Greek Physician, Hippocrates, known as the father of medicine "Let food be thy medicine" centuries ago upholds the importance of good nutrition through healthy food choices. The emphasis is on positive dietary aspects in prevention of diseases and enhancement of the health of an individual. This recognition of the correlation between health and nutrition paved the way for evolution of "nutraceuticals" [1]. A survey conducted in UK, Germany and France where consumers rated diet higher than exercise or hereditary factors to achieve a good health led to the concept of "nutraceuticals". (Pandey et al) The broad term nutraceuticals was coined by Stephen De Felice, founder and chairman of the foundation for innovation medicine, Cranford, New Jersey in 1989 (Maddi et al 2007).The term is a hybrid of 'nutrition' and 'pharmaceuticals' and defined by Stephen de Felice as 'a food (or a part of food) that provides medical or health benefits, including the prevention and or treatment of a disease' [2].

Some other terms used in this context are functional foods and dietary supplements. Functional foods may be defined as food products that are to be taken as part of the usual diet in order to have beneficial effects that go beyond basic nutritional function. Here food is being cooked or prepared using "scientific intelligence" with or without knowledge of how or why it is being used [3]. According to Dietary Supplement, Health and Education Act (DSHEA); dietary supplements are products intended to supplement the diet that bears or contains one or more of the following dietary ingredients: a vitamin, a mineral, a herb or other botanical, an amino acid, a dietary substance for use by man to supplement the diet by increasing the total daily intake, or a concentrate, metabolite, constituent, extract, or combinations of these ingredients [4]. A clear demarcation is not available among these terms. Nutraceuticals can be classified on the basis of their natural sources, pharmacological conditions, or as per chemical constitution of the products etc. On the basis of natural source, it can be classified as the products obtained from plants, animals, minerals, or microbial sources. Based on Pharmacological conditions, it is classified for use in cardiac disease, allergies etc and are classified as digestive enzymes, vitamins etc. based on their chemical composition [4]. 
India has recently passed the regulations for nutraceuticals under 'Food Safety and Standard Act 2006', a modern integrated food law to serve as a single reference point in relation to regulation of food products including nutraceutical, dietary supplements and functional food. Nutraceuticals differ from Pharmaceuticals by not having patent protection. Most of the nutraceutical products marketed are potential nutraceuticals and have not undergone any cinical trials [5]. Most of the synthetic drugs possess properties pertaining to toxicities. Nutraceutical compounds, herbal nutraceuticals have been investigated for their potential in combating the tox ic effects of toxins and other medications. Most of the plants possess antioxidant activity and other than various specific ways to combat toxins and synthetic drugs, they generally may reduce their toxicity by reduction of oxidative stress. Nowadays, nutraceuticals are receiving high interest due to their potential nutritional and safety profile, other than therapeutic capability [5]

Mutually conflicting evidence makes it difficult to recommend or preclude the use of nutraceuticals which are increasingly being used in day to day practice. There is a dearth of studies outlining the use and prescription of nutraceuticals in the Indian population. Hence we conducted a pilot study aims to assess the knowledge, attitude and practices regarding nutraceuticals among registered medical practitioners of a tertiary care teaching hospital in Dakshina kannada district of South India.

\section{Materials And Methods}

This pilot study was conducted as a cross sectional, questionnaire based study. The study was initiated after obtaining approval from the Institutional Ethics Committee of A. J. Institute of Medical Sciences \& Research Centre, Mangaluru, dated 29 January 2016 (Ethics clearance no. AJEC/REV/09/2016)

\section{Duration of study}

The pilot study was conducted in February 2016 for a period of one month.

\section{Study population and design:}

The study was conducted using a self structured, validated questionnaire based design. Study was conducted among 50 participants as part of pilot study. Interns, alternative medical practitioners were excluded from the study.

\section{The questionnaire:}

The questionnaire was given to the medical practitioners along with information sheets about purpose of the survey. The questionnaire was self-structured and pretested. Some questions were reframed, depending on the answers and suggestions. The questionnaire was divided into four sections. Section I consisted of the demographic details of the participants. The age, gender and years of experience were asked. Name and other personal details were optional. The sections II, III and IV had a total of 18 questions. Some of the questions had multiple choices. Section II focused on the knowledge based questions whereas three and four were related to attitude and practice regarding nutraceuticals, respectively.

\section{The study:}

50 registered medical practitioners were approached from different departments in the hospital and were explained in detail regarding the purpose of the study. The informed consent document and questionnaire was handed out to the practitioner and were requested to fill the form. The completed forms were then assessed.

\section{Results}

A total of 50 questionnaires were completed and returned by the approached registered medical practitioners. The forms were analysed and expressed in percentages. The sum total of percentages is not always $100 \%$ since the questionnaire had questions with multiple options to choose from.

Demographic details of the pilot study participants are as follows: As shown in "table 1"

\begin{tabular}{|l|l|}
\hline Variable & $\begin{array}{c}\text { Percentage }(\%) \\
(\mathrm{n}=50)\end{array}$ \\
\hline Gender & \\
Male & $40(80)$ \\
Female & $10(20)$ \\
\hline Years of & $20(40)$ \\
experience & $13(26)$ \\
$<5$ 5-10yrs & $11(22)$ \\
$11-20 \mathrm{yrs}$ & $07(14)$ \\
$>20 \mathrm{yrs}$ & \\
\hline
\end{tabular}


Table 1: Demographic details of the study participants expressed in percentages Knowledge:

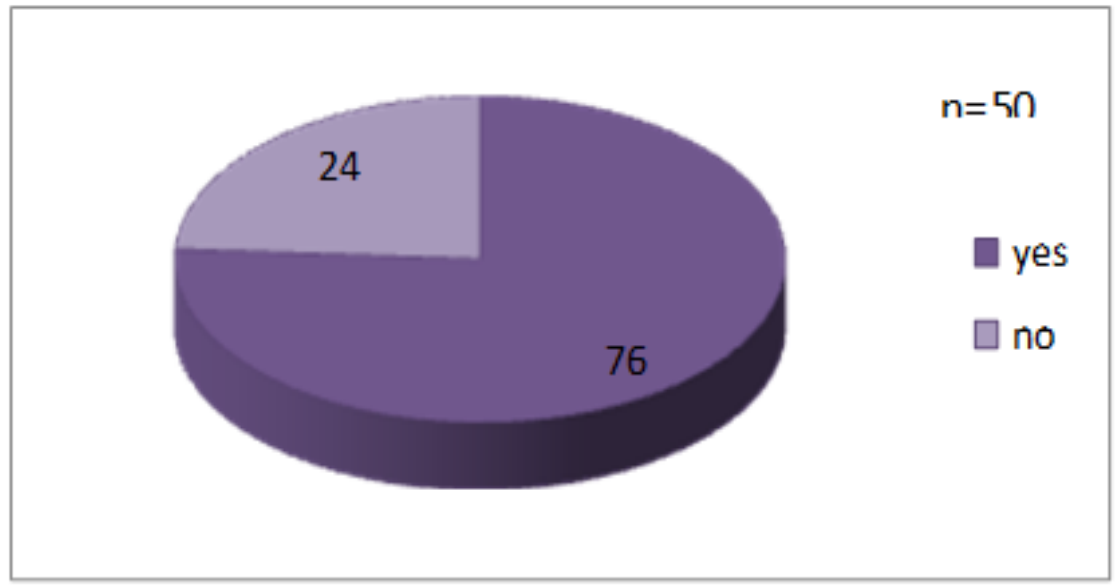

Figure 1: Pie chart showing number of respondants who were aware of the term nutraceuticals

$76 \%$ of the study participants were aware of the term 'nutraceuticals'.(Fig 1) $46 \%$ of the respondants said that nutraceuticals are not same as herbal medicines wheras $18 \%$ of them said that it is the same. When questioned regarding the beneficial effecs of nutraceuticals $72 \%$ of the study participants said that nutraceuticals help in preventing the disease and has lesser side effects. $18 \%$ among the participants felt it is cost effective. The patient compliance was said to be $50 \%$ whereas $26 \%$ participants said otherwise.

\section{Attitude:}

$78 \%$ of the respondents felt that sufficient information is not available to them regarding efficacy and safety of nutraceuticals. Most of them said that sufficient information is not available regarding use of nutraceuticals in special groups like paediatric and geriatric age group. (Fig 2)

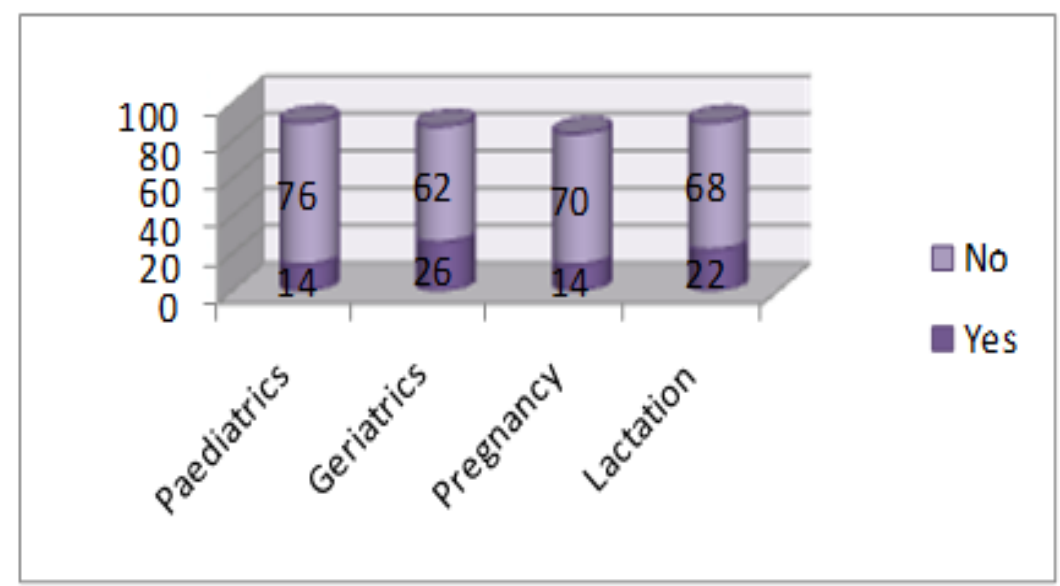

Figure 2: Column chart showing availability of information regarding nutraceuticals in special groups.

The percentage of respondents who said that nutraceuticals should be sold only on prescription of a registered medical practioner and all of them felt that the manufacture and sale of nutraceuticals should be monitored by a regulatory body.

\section{Practice:}

Among the 50 respondents, 54\% prescribe nutraceuticals in their daily practice. They generally preferred to prescribe in the elderly age group, i.e., 24\% preferred nutraceuticals in the age group of 46 to 64 years and $9 \%$ for age groups above 65 years. $4 \%$ said they have seen adverse effects with nutraceutical use. $23 \%$ of the respondants procured information regarding use of nutraceuticals from pharmaceutical company respondents whereas 22\% obtained them from Drug package inserts. (Fig 3) 


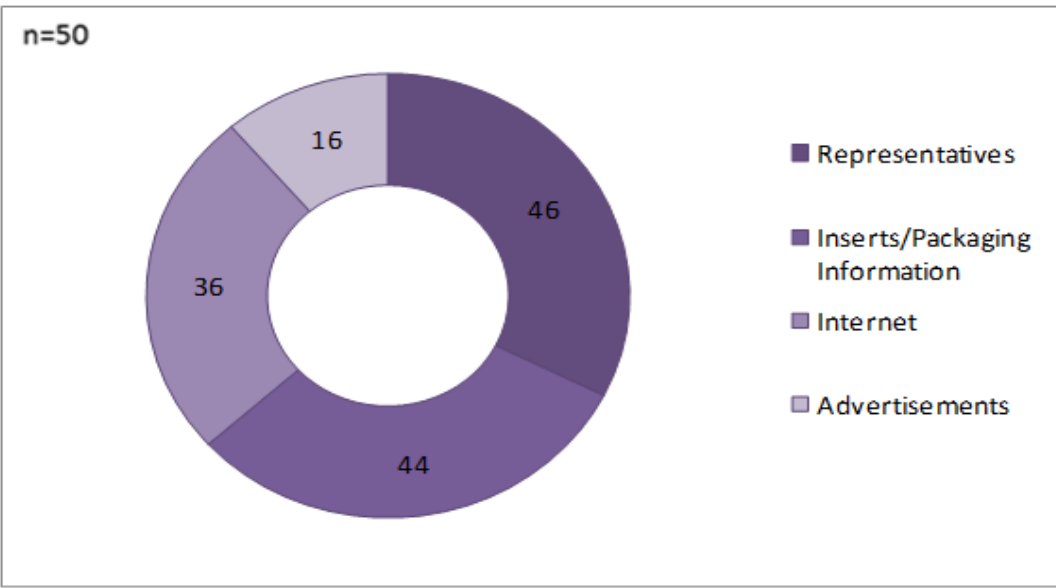

Figure 3: Chart showing procurement of information regarding nutraceuticals by practitioners.

\section{Discussion}

There is a growing interest in the relationship between diet and health that has led to the increased demand for information on nutraceuticals. This increased interest has been aided by advances in science and technology, increasing health care costs, changes in food laws affecting label, and product claims and aging population in various countries. Along with offering treatment, use of nutraceuticals favours a preventive treatment model as well [6]. In our study, $76 \%$ of the practitioners were aware of the term nutraceuticals. Since further answering would not be possible if they were not aware of the term, a brief introduction with few examples was given in the covering page of the informed consent form regarding nutraceuticals.

$38 \%$ of the respondants said they did not know if herbal drugs and nutraceuticals were the same. Most of the respondents said that nutraceuticals help in disease prevention and has lesser side effects. Only $4 \%$ of the respondants have seen adverse effects with use of nutraceuticals like gastritis and constipation. Since the details regarding adverse effects, like name of the drug used or concurrent medication use was not asked in the questionnaire, the causality assessment is not known. $78 \%$ of the participants feel more information regarding nutraceutical use like dosage, formulation, adverse effects, drug interaction is necessary. Details of use in special population like paediatrics and pregnancy is also lacking which aid in prescribing drugs. All the participants thought it is necessary to manufacture and sell these drugs under regulatory bodies. Nutraceuticals have a promising role in the promotion of human health and disease prevention. So health professional, nutritionists, and regulatory toxicologist should join hands to plan appropriate regulation that provides benefit to mankind. Hence the implementation of regulatory body is necessary to standardize the nutraceutical industry ${ }^{4}$

This pilot study was conducted and the questionnaire was validated with 50 respondents. A larger scale study using the questionnaire will effectively describe the status of nutraceuticals pertaining to clinical practice in India.

\section{Conclusion}

Nutraceuticals may be beneficial to our health but the benefits and harmful effects have to be studied extensively. There are lot of challenges in this new category of health products that lies between food and drugs. Nutraceuticals are increasingly being prescribed by medical practitioners but there is need for further study to analyse the attitude and practices towards them to provide ultimate benefit to mankind.

\section{Acknowledgements}

The authors would like to thank the Practitioners who participated in the study in spite of their busy schedules.

\section{References}

[1]. Ramaa CS, Shirode AR, Mundada AS, Kadam VJ. Nutraceuticals - Role of nutraceuticals in human health-An emerging trend in the treatment and prevention of cardiovascular diseases Curr Pharm Biotechnol. 2006 Feb;7(1):15-23.

[2]. Das L, Bhaumik E, Raychaudhuri U, Chakraborty R. Role of nutraceuticals in human health. Journal of food science and technology. 2012;49(2):173-183. doi:10.1007/s13197-011-0269-4.

[3]. Kalra EK. Nutraceutical - Definition and introduction. AAPS PharmSci. 2003;5:E25

[4]. Chauhan B, Kumar G, Kalam N, Ansari SH. Current concepts and prospects of herbal nutraceutical: A review. Journal of Advanced Pharmaceutical Technology \& Research. 2013;4(1):4-8. doi:10.4103/2231-4040.107494.

[5]. Nasri H, Baradaran A, Shirzad H, Rafieian-Kopaei M. New Concepts in Nutraceuticals as Alternative for Pharmaceuticals. International Journal of Preventive Medicine. 2014;5(12):1487-1499.

[6]. Balhara YP, Verma R. Psychoactive nutraceuticals. J Med Nutr Nutraceut 2012;1:27-36 